\title{
Espiroquetosis intestinal humana: serie clínica y revisión de la literatura
}

\author{
Carlo Lozano, Leonardo Arellano y Pamela Yaquich
}

\section{Human intestinal spirochetosis: clinical series and literature review}

Introduction: Human intestinal spirochetosis (HIE) is defined as colonization by spirochetes of the large intestine. Is associated with chronic diarrhea. The incidence and prevalence ranges from $0.4 \%$ to $12 \%$. Objective: To determine the prevalence of HIE in the Salvador's Hospital, between 2003 and 2008 in patients with a history of chronic diarrhea and without abnormalities in colonoscopy, in 2 separate groups: patients with and without a history of HIV infection. Material and Methods: Retrospective morphology evaluation of the large bowel endoscopic biopsies to the selected groups. Results: We reviewed 115 biopsies, 98 were from HIV-negative and 17 HIV from positive patients. Two cases of intestinal spirochetosis were detected, both HIV negative, with a prevalence of 1.7\%. Comment: The prevalence of HIE is similar to that reported in Western countries. Population studies are needed to determine the real epidemiological impact in our environment.

Key words: Spirochetosis, colon, diarrhea.

Palabras clave: Espiroquetosis, colon, diarrea.

\section{Introducción}

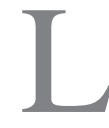

a espiroquetosis intestinal humana (EIH), descrita en 1967 por Harland y Lee, se define como la

colonización del colon y recto por espiroquetas ${ }^{1-4}$.

Es causa frecuente y conocida de diarrea en aves de corral, perros y cerdos, asociándose a grandes pérdidas económicas en la producción de alimentos. En humanos son las especies Brachyspira aalborgi y Brachyspira pilosicoli las más frecuentes, pero aún se discute el real rol patógeno que poseen, si bien se asocian a diarrea crónica. En humanos la incidencia y prevalencia varían desde 0,4 a $12 \%{ }^{5-8}$, describiéndose en general, mayor frecuencia en hombres, particularmente homosexuales, pacientes infectados por VIH y zonas de pobreza. Estas cifras se modifican sustancialmente según la región geográfica y la población evaluada. No hay registros epidemiológicos en nuestro medio. Presentamos una serie clínica local con dos casos de espiroquetosis intestinal.

\section{Material y Método}

El objetivo principal fue determinar la frecuencia de EIH en el Hospital Del Salvador, institución que atiende sólo adultos, entre enero del año 2003 y octubre del año 2008, a través de la revisión de las biopsias de intestino grueso realizadas a pacientes con antecedentes clínicos de diarrea crónica sin causa determinada y colonoscopia sin hallazgos patológicos. Los casos fueron revisados en forma simultánea por dos médicos anátomo-patólogos, realizándose en los casos sospechosos tinciones histoquímicas de Warthin Starry (WS), ácido peryódico de Schiff (PAS), Grocott (CR) y tinción de Gram para confirmar el diagnóstico, según las propiedades tintoriales de las espiroquetas, es decir, positivas a las tinciones de PAS, GC y WS, negativas a la tinción de Gram. Se realizó, según la disponibilidad del examen, una tinción por medio de inmunohistoquímica con anticuerpo policlonal anti Treponema pallidum debido a su reacción cruzada con las espiroquetas intestinales. Los casos fueron separados en dos grupos, correspondientes a pacientes con y sin antecedentes clínicos de infección por VIH, debido a la asociación que sugiere la literatura científica. La información clínica de los dos pacientes que resultaron positivos se obtuvo tanto del informe de la endoscopia como de su ficha médica.

Se definió como espiroquetosis intestinal la presencia de una banda basófila de elementos bacterianos filamentosos, dispuesta sobre el epitelio, la que se interrumpía en relación a las células caliciformes (Figura 1) y presentaba tinción positiva para WS, GC y PAS (Figura 2), siendo negativas para la tinción de Gram.

\section{Resultados}

En el período señalado, se revisaron 115 biopsias que cumplían los criterios de selección, 98 del grupo de pacientes seronegativos para VIH y 17 en el grupo de pacientes infectados por VIH. Se detectaron cinco casos

\author{
Hospital del Salvador, \\ Santiago de Chile. \\ Servicio de Anatomía Patológica \\ (CL, LA). \\ Servicio de Gastroenterología (PY). \\ Recibido: 4 de mayo de 2011 \\ Aceptado: 8 de mayo de 2012 \\ Correspondencia a: \\ Carlo Lozano \\ carlo.lburgos@gmail.com
}


Figura 1. Mucosa de intestino grueso con arquitectura conservada y escaso infiltrado inflamatorio mononuclear en la lámina propia, sin signos de infección aparente. HE x 40.

Figura 2. Mucosa de intestino grueso con banda filamentosa supraepitelial, que se interrumpe en relación a las células caliciformes. HE x 200.

Figura 3. Tinción inmunohistoquímica con anticuerpo anti- Treponema pallidum, positiva en las espiroquetas, las que invaden la lámina propia. HE $\times 100$. sospechosos, de los cuales finalmente se confirmaron dos casos de EIH, ambos en individuos seronegativos para VIH, correspondientes a un hombre de 62 años residente en Santiago, con antecedentes de cáncer de próstata operado el año 2007, sin evidencia de recidiva en el último control, y una mujer de 66 años, residente en la Isla de
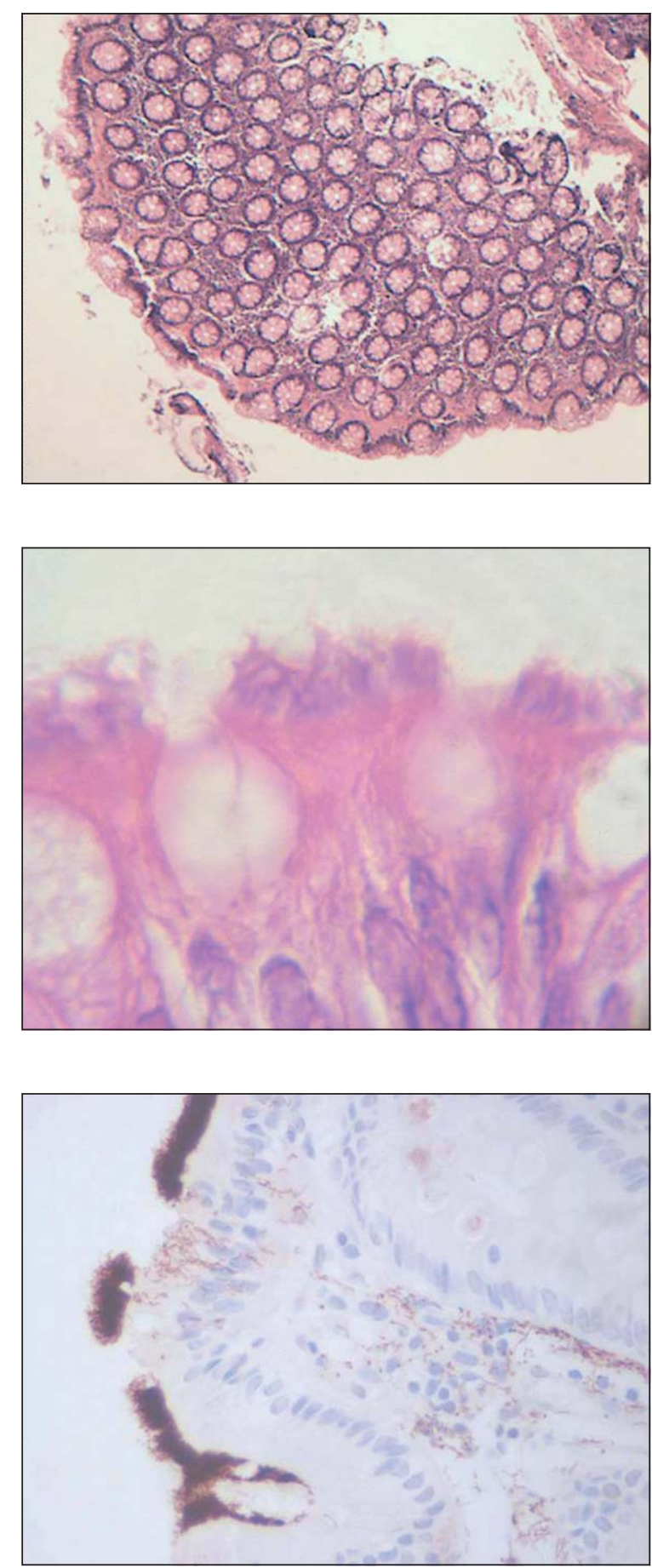

Pascua, sin antecedentes mórbidos relevantes. En el primer caso se realizó además tinción inmuno-histoquímica con anticuerpo anti- T. pallidum que resultó intensamente positiva en el borde filamentoso bacteriano (Figura 3). Los hallazgos clínicos y morfológicos de ambas biopsias se resumen en la Tabla 1.

\section{Discusión}

Las espiroquetas son bacterias anaeróbicas de tipo filamentosas, negativas a la tinción de Gram y positivas al PAS y tinciones de plata como CR y WS, considerándose esta última como la más específica. Las que en mayor proporción comprometen el intestino grueso en los casos de espiroquetosis humanas son del género Brachyspiras, tanto B. aalborgi como B. pilosicoli. En general, estas bacterias son causa frecuente de diarrea en aves de corral, pero también se describen en perros y cerdos, lo que ha permitido plantear que estos se comportan como reservorios para la transmisión a humanos ${ }^{10-13}$, si bien aún no se establece con claridad la forma del contagio. El mecanismo que causa la enfermedad es también controversial; sin embargo, se ha establecido que las espiroquetas tiene un efecto citopático asociado al aplanamiento y destrucción de microvellosidades y la inducción de cambios en el citoesqueleto celular ${ }^{14}$.

Su distribución epidemiológica es variable; en general hay reportes sobre poblaciones específicas sin una clara tendencia, salvo la predilección por el género masculino. Se ha reportado una prevalencia aumentada en subgrupos, tales como hombres homosexuales, pacientes portadores del VIH, zonas de pobreza, disponibilidad de agua potable, pacientes críticos en falla orgánica múltiple, y co-existencia con infección gonocóccica, entre otros, lo que varía según la región estudiada ${ }^{8,15-20}$.

En cuanto a su expresión clínica, los síntomas asociados más comunes son diarrea y dolor abdominal, también la alternancia entre diarrea y constipación o la distensión abdominal, en general síntomas inespecíficos ${ }^{15}$. En las colonoscopias no se encuentran por lo general alteraciones de la mucosa y por tanto, el grupo que debe ser evaluado por el patólogo con más precaución en la biopsia, es aquel con diarrea crónica y colonoscopia normal ${ }^{5,6}$. En relación al chancro sifilítico, particularmente en pacientes homosexuales y pacientes seropositivos para VIH, la biopsia de estas lesiones puede demostrar la presencia de espiroquetas con características similares, tanto morfológicas como inmunohistoquímicas, por lo que el diagnóstico debe establecerse considerando que esta entidad se presenta como una lesión indurada rectal que más bien simula una neoplasia, cursa con serología positiva y a veces con manifestaciones extraintestinales de sífilis ${ }^{21,22}$.

El diagnóstico de la espiroquetosis intestinal humana 


\section{Experiencia Clínica}

Tabla 1. Características clínicas e histopatológicas de dos casos de espiroquetosis intestinal

\begin{tabular}{|lll|}
\hline & Tabla 1. Características clínicas e histopatológicas de dos casos de espiroquetosis intestinal \\
\hline Edad (años) & Paciente 1 & Paciente 2 \\
\hline Sexo & 62 & 66 \\
\hline Presentación clínica & Masculino & Femenino \\
\hline Compromiso mucoso & Diarrea crónica & Diarrea crónica \\
\hline Arquitectura de la mucosa & Difuso & Focal \\
\hline Compromiso por espiroquetas & Borde epitelial y lámina propia & Normal \\
\hline Infiltrado inflamatorio & Escaso, mononuclear de predominio linfoide en la lámina propia & Escaso, mononuclear de predominio linfoide en la lámina propia \\
\hline
\end{tabular}

se sospecha en la biopsia por la presencia de una banda supraepitelial basófila y filamentosa, que se interrumpe en las células caliciformes, al contrario de la mucina con la que se confunde habitualmente. Su presencia amerita un estudio complementario, particularmente con la tinción histoquímica de WS, la más específica para este tipo de bacterias y, si es posible, la confirmación con una tinción con anticuerpo policlonal anti T. pallidum, ya sea por inmunohistoquímica o por inmunofluorescencia, la que resulta positivo debido a que existe reacción cruzada con el género Brachyspira ${ }^{2,3}$. Los cultivos de deposiciones son complejos y positivos sólo en 50\% de los casos detectados por reacción de polimerasa en cadena (RPC) 2,3,4,22-24, razón por la cual se sugiere como método diagnóstico complementario en muestras de deposiciones, ya sea para un caso individual como en estudios poblacionales en humanos y animales, la determinación de la porción específica 16SARN ribosomal o el gen de la NADH oxidasa, amplificadas por RPC.

Con referencia al tratamiento, se debe considerar que en estudios poblacionales la mayoría de los casos son asintomáticos; no obstante, el uso de antimicrobianos como metronidazol ha demostrado ser útil en la remisión de los síntomas, razón por la cual se sugiere que esta entidad sea considerada y tratada como una enfermedad, recomendándose además un control con biopsia posterior al tratamiento para confirmar la erradicación ${ }^{6,15-17}$.

Finalmente, concluimos que la espiroquetosis intestinal es una entidad asociada a diarrea crónica con colonoscopia normal, al parecer de una baja prevalencia en nuestro medio. En esta serie los dos casos detectados correspondieron al grupo de pacientes sin antecedentes de infección por $\mathrm{VIH}$, lo que es discordante con los resultados publicados, quizás debido a una muestra reducida de casos o al uso de antimicrobianos por otras infecciones más frecuentes en estos pacientes. El diagnóstico es fundamentalmente, y en la mayoría de los casos, sospechado y confirmado por el patólogo, el que debe considerar esta posibilidad diagnóstica en la biopsia de un paciente con diarrea crónica. Por último, creemos fundamental ampliar este informe con estudios epidemiológicos de prevalencia de estas especies de espiroquetas en deposiciones, tanto en nuestra población como en animales domésticos y de consumo humano.

\section{Resumen}

Introducción: La espiroquetosis intestinal humana (EIH) se define como la colonización del intestino grueso por espiroquetas. Se asocia a diarrea crónica. Su incidencia y prevalencia van desde 0,4 a 12\% Objetivo: Determinar la prevalencia de EIH en el Hospital Del Salvador, de Santiago, Chile, entre los años 2003 y 2008, en pacientes con antecedentes clínicos de diarrea crónica y colonoscopia sin hallazgos patológicos, separados en dos grupos: pacientes con y sin antecedentes de infección por VIH. Material y Método: Evaluación morfológica retrospectiva de las biopsias endoscópicas de intestino grueso de los grupos seleccionados. Resultados: Se revisaron 115 biopsias, 98 correspondieron a pacientes sin infección por VIH y 17 a pacientes seropositivos para VIH. Se detectaron dos casos de espiroquetosis intestinal, ambos en pacientes sin infección por VIH, con una prevalencia de 1,7\%. Comentario: La prevalencia de EIH es similar a la publicada en países occidentales. Se requieren estudios poblacionales para determinar el real impacto epidemiológico en nuestro medio.

\section{Referencias bibliográficas}

1.- Mikosza A S, Hampson D J. Human intestinal spirochetosis: Brachyspira aalborgi and/or Brachyspira pilosicoli? Anim Health Res Rev 2001; 2: 1001-10. Review.
2.- Harland W A, Lee F D. Intestinal spirochetosis. Br Med J 1967; 3: 718-9.

3.- De Brito T, Sandoval M P, Silva A G, Saad R C, Colaiacovo W. Intestinal spirochetosis: first cases reported in Brazil and the use of immunohistochemistry as an aid in histopathological diagnosis. Rev Inst Med Trop Sao Paulo 1996; 38: 45-52.

4.- $\quad$ Brooke C J, Riley T V, Hampson D J. Evaluation of selective media for the isolation of Brachyspira aalborgi from human faeces. J Med Microbiol 2003; 52 (Pt 6): 509-13. 
5.- Tanahashi J, Daa T, Gamachi A. Human intestinal spirochetosis in Japan; its incidence, clinic pathologic features, and genotypic identification. Mod Pathol 2008; 21: 76-84.

6.- Esteve M, Salas A, Fernández-Bañares F. Intestinal spirochetosis and chronic watery diarrhea: clinical and histological response to treatment and long-term follow up. J Gastroenterol Hepatol 2006; 21: 1326-33.

7.- Peruzzi S, Gorrini C, Piccolo G. Human intestinal spirochaetosis in Parma: a focus on a selected population during 2002-2005. Acta Biomed 2007; 78: 128-32.

8.- Margawani K R, Robertson I D, Brooke C J, Hampson D J. Prevalence, risk factor and molecular epidemiology of Brachyspira pilosicoli in humans on the island of Bali, Indonesia. J Med Microbiol 2004; 53: 325-32.

9.- Macaigne G, David F, Boivin J F. Colorectal spirochetosis, a possible but inusual cause of chronic diarrhea. Presse Med 2002; 31: 458-61.

10.- Feberwee A, Hampson D J, Phillips N D. Identification of Bachyspira hyodysenterie and pathogenic Bachyspira species in chickens from laying flocks with diarrhea or reduced production or both. J Clin Microbiol 2008; 46:
593-600.

11.- Stephens C P, Hampson D J. Intestinal spirochete infections of chickens: a review of disease associations, epidemiology and control. Anim Health Res Rev 2001; 2: 83-91.

12.- Oxberry S L, Hampson D J. Colonisation of pet shop puppies with Brachyspira pilosicoli. Et Microbiol 2003; 93: 167-74.

13.- Barcellos D E, Mathiesen M R, de Uzeda M. Prevalence of Brachyspira species isolated from diarrhoeic pigs in Brazil. Vet Rec 2000; 146: 398-400.

14.- Rodgers F G, Rodgers C, Shelton A P. Proposed pathogenic mechanism for the diarrhea associated with human intestinal spirochetes. Am Clin Pathol 1986; 86: 679-82.

15.- Weisheit B, Bethke B, Stolte M. Human intestinal spirochetosis: analysis of the symptoms of 209 patients. Scand J Gastroenterol 2007: 42: 1422-7.

16.- Knofp B, Bethke B, Stolte M. Human intestinal spirochetosis. Pathologe 2003; 24: 192-5.

17.- Van Mook W N, Koek G H, van der Ven A J. Human intestinal spirochetosis: any clinical significance?. Eur J Gastroenterol Hepatol 2004; 16: 83-7.

18.- Körner M, Gebbers J O. Clinical significance of human intestinal spirochetosis a morphologic approach. Infection 2003; 31: 341-9.

19.- Käsbohrer A, Gelderblom H R, Arasteh K. Intestinal spirochetosis in HIV infection: prevalence, isolation and morphology of spirochetes. Dtsch Med Wochenschr 1990; 115: 1499-506.

20.- Surawicz C M, Roberts P L, Rompalo A. Intestinal spirochetosis in homosexual men. Am J Med 1987; 82: 587-92.

21.- Febbraro I, Manetti G, Balestrieri P. Rectal cancer or rectal chancre? Beware of primary syphilis. Dig Liver Dis 2008; 40: 579-81.

22.- Quinn T C, Luckerhart S A, Goodell S. Rectal mass caused by Treponema pallidum: confirmation by immunofluorescent staining. Gastroenterology 1982; 82: 135-9.

23.- Mikosza A S, La T, Margawani K R, Brooke C J, Hampson D J. PCR detection of Brachyspira aalborgi and Brachyspira pilosicoli in human faeces. FEMS Microbiol Lett 2001; 197: 167-70.

24.- Phillips N D, La T, Hampson D J. Across-sectional study to investigate the occurrence and distribution of intestinal spirochaetosis in three flocks of laying hens. Vet Microbiol 2005; 105: 189-98. 